\title{
Violência infanto-juvenil intrafamiliar e doméstica: o impacto do distanciamento social e a importância da conscientização em meio à pandemia de COVID-19
}

\author{
Domestic and intra-family violence against children and adolescents: the impact of social \\ distance and the importance of awareness amid the COVID-19 pandemic \\ Violencia infantil-juvenil intrafamiliar y doméstica: impacto del distanciamiento social y la \\ importancia de la concienciación en medio de la pandemia del COVID-19
}

Ana Carolina Mathias Santa Rita ${ }^{1}$, Ana Clara Guimarães de Castro ${ }^{1}$, Bárbara Nogueira Roberti ${ }^{1}$, Isabel Drummond Teixeira ${ }^{1}$, Lucca Batista Rocha de Menezes ${ }^{1}$, Marina Brettas Tavares ${ }^{1}$, Victória Cristine Araujo Dias ${ }^{1 *}$, Vitor Augusto Lima do Vale ${ }^{1}$, Cristiane de Freitas Cunha Grillo².

\section{RESUMO}

Objetivo: Analisar e difundir o conhecimento acerca do aumento da violência infanto-juvenil, em função da pandemia de Coronavírus. Revisão bibliográfica: A partir do contexto da pandemia de Covid-19, no qual o distanciamento social apresenta-se como a principal medida de proteção para a população, a violência familiar/doméstica infanto-juvenil, que já era prevalente, intensificou em todo o mundo, inclusive no Brasil. Nesse contexto, devido ao ambiente domiciliar, muitas vezes conturbado e estressante, são evidenciados quadros de maior irritabilidade tanto nos agressores, quanto nas crianças e nos adolescentes, o que, consequentemente, acentua os conflitos. Além disso, a maior permanência no ambiente doméstico, priva a supervisão das vítimas por terceiros, como educadores e profissionais da saúde, o que contribui para o aumento da subnotificação da violência. Desse modo, é necessário conhecimento das formas de cuidado e atenção aos sinais de violência, com destaque para o papel fundamental da denúncia, realizada principalmente por parentes próximos e vizinhos. Considerações finais: Conclui-se que a identificação precoce da violência infanto-juvenil propicia a manutenção da integridade física e mental, imprescindíveis para garantir um adequado desenvolvimento do indivíduo.

Palavras-chave: Violência doméstica, Pandemias, Quarentena, Maus-tratos infantis.

\section{ABSTRACT}

Objective: Analyze and disseminate knowledge about the increase in violence against children and adolescents, due to the Coronavirus pandemic. Bibliographic review: From the context of the COVID-19 pandemic, in which social distance is the main protective measure for the population, domestic and intra-family violence against children and adolescents, which was already prevalent, intensified throughout the world, including in Brazil. In this context, due to the home environment, which is often troubled and stressful, situations of greater irritability are evidenced both in the aggressors, and in the children and adolescents, which, consequently, accentuates the conflicts. In addition, the greater permanence in the domestic environment deprives the victims of supervision by third parties, such as educators and health professionals, which contributes to the underreports of violence. Thus, it is necessary to know the forms of care and attention to signs of violence, with emphasis on the fundamental role of the complaint, carried out mainly by close relatives and neighbors. Final considerations: It is concluded that the early identification of violence against children and adolescents promotes the maintenance of physical and mental integrity, which are essential to ensure an adequate development of the individual.

Key words: Domestic violence, Pandemics, Quarantine, Child abuse.

\footnotetext{
${ }^{1}$ Pontifícia Universidade Católica de Minas Gerais (PUC-MG), Betim - MG.

*E-mail: victoria.araujo.dias@gmail.com

2 Universidade Federal de Minas Gerais (UFMG), Belo Horizonte - MG.
}

SUBMETIDO EM: 7/2020

ACEITO EM: 8/2020

PUBLICADO EM: 10/2020 


\section{RESUMEN}

Objetivo: Analizar y difundir el conocimiento acerca del aumento de la violencia infantil-juvenil, debido a la pandemia del Coronavirus. Revisión bibliográfica: A partir del contexto de la pandemia del COVID-19, en el cual el distanciamiento social se presenta como la principal medida de protección para la población, la violencia familiar/doméstica, infantil-juvenil, que ya era predominante, se ha intensificado en todo el mundo, incluso en Brasil. En este contexto, en el seno de un ambiente doméstico, muchas veces complicado y estresante, se han detectado cuadros de mayor irritabilidad tanto en los agresores, como en los niños y adolescentes, lo que, consecuentemente, acentúa los conflictos. Además, la mayor permanecía en el domicilio familiar, dificulta la supervisión de las víctimas por terceros, como educadores y profesionales sanitarios, lo que implica un descenso de las notificaciones de violencia doméstica. De esta manera, se hace necesario un mayor conocimiento de las formas de cuidado y atención ante las señales de violencia, destacando el papel fundamental de las denuncias, realizadas principalmente por familiares próximos y vecinos. Consideraciones finales: Se concluye que la identificación prematura de la violencia infantil-juvenil implica el soporte de la integridad física y mental, imprescindible para garantizar el adecuado desarrollo del individuo.

Palabras clave: Violencia doméstica, Pandemias, Cuarentena, Maltrato a los niños.

\section{INTRODUÇÃO}

O artigo 196 da Constituição da República Federativa do Brasil (CRFB) prevê o acesso universal e igualitário de todos à saúde. Dessa forma, percebe-se que qualquer indivíduo, independentemente da faixa etária, tem direito de receber atenção em saúde e de ter assegurado acesso holístico ao cuidado (BRASIL, 1998).

A definição da palavra "saúde", elaborada pela Organização Mundial da Saúde (OMS), transcende a ausência de doença, e abrange o estado pleno de bem-estar físico, mental e social (SILVA MJS, et al., 2019). Contudo, sabe-se que esse conceito é muitas vezes negligenciado, visto que muitos jovens encontram-se em posição de comprometimento do estado de saúde, fato esse que viola, também, o Estatuto da Criança e do Adolescente (ECA) (BRASIL, 2019a).

Dessa forma, o ECA determina que todo caso de suspeita ou de confirmação de violência contra criança deve ser obrigatoriamente informado ao Conselho Tutelar, sem que isso impeça as outras providências legais (BRASIL, 2019a). Mesmo que a subnotificação dos episódios de violência seja uma realidade, acarretando uma redução aparente do número real de casos, percebe-se ainda a existência de muitas vítimas. De acordo com o boletim epidemiológico emitido pelo Ministério da Saúde, em 2017 foram registradas 307.367 notificações de violência interpessoal/autoprovocada no Brasil, sendo que 46,5\% envolviam adolescentes (1019 anos) (BRASIL, 2019b).

Em estudo, os pais foram apontados como os maiores responsáveis pela violência contra crianças e adolescentes, principalmente entre aqueles com faixa etária menor ou igual a 5 anos (NUNES AJ e SALES MCV, 2016). Esse fato explicita um paradoxo nos casos de violência intrafamiliar e doméstica, uma vez que a família, socialmente apresentada como refúgio e abrigo, se coloca no papel de agressora da criança e do adolescente por quem deveria ser responsável.

Dentro desse contexto, acredita-se que a violência e a vulnerabilidade são ainda maiores para esses indivíduos durante os períodos de ausência escolar decorrentes de emergências de saúde (CLUVER L, et al., 2020). Dessa forma, tem-se noticiado aumento exorbitante dos casos de violência doméstica e intrafamiliar contra crianças e adolescentes tendo em vista o cenário mundial, e principalmente nacional, provocado pela pandemia de Coronavírus.

No estado do Paraná, por exemplo, identificou-se um aumento de $15 \%$ nos relatos de violência doméstica, incluindo casos praticados contra crianças e adolescentes recebidos pela Polícia Militar (PM) no primeiro final de semana do distanciamento social; situações semelhantes foram identificadas no Ceará, Pernambuco e São Paulo (MARQUES ES, et al., 2020). 
Essa condição é justificada pelo fato de que, quando jovens vulneráveis são restritos a nada além de contato com a família por um longo período, há condições perfeitas para uma falha na proteção dos menores, visto que, sem o convívio regular com professores e com profissionais da área da saúde, perdem-se as oportunidades rotineiras de identificar sinais ou narrativas de ameaça à segurança (GREEN P, 2020).

Dessa forma, o número de crianças e adolescentes vítimas da violência permanecem, elevado, visto que o apoio e a proteção, que deveriam ser constantes, tornaram-se limitados e, até mesmo, inexistentes. Assim, essa crescente incidência nos índices de violência doméstica nesse período de contenção da pandemia torna ainda mais urgente essa discussão.

O objetivo deste trabalho concentra-se, portanto, em difundir esse conhecimento, pois, apesar de altamente prevalente, a violência domiciliar e intrafamiliar infanto-juvenil ainda é pouco discutida e, muitas vezes, negligenciada. Por fim, sabe-se que os danos provocados por essas agressões perpetuam-se no desenvolvimento biopsicossocial e impactam na formação do indivíduo, o que reforça a importância da intervenção quanto a essas questões o mais precocemente possível.

\section{REVISÃO BIBLIOGRÁFICA}

Violência consiste, segundo a OMS, no uso ou ameaça intencional de força física ou poder contra si mesmo ou outras pessoas, capaz de provocar ferimentos, prejuízos psicológicos, de desenvolvimento ou de privação, e até mesmo morte (DAHLBERG LL e KRUG EG, 2007).

Associado a isso, sabe-se que a violência intrafamiliar tem sido a forma mais prevalente de violação de direitos, sendo está caracterizada por prejudicar o direito integral de desenvolvimento de outro membro da família no que tange ao espaço físico e às relações estabelecidas (STEFANINI JR, et al., 2019).

Destaca-se ainda que essas agressões podem ser cometidas dentro ou fora do domicílio por pais ou indivíduos com função parental, independente dos laços sanguíneos. Outro tipo comum de agressão, frequentemente relacionado à anterior, é a violência doméstica, a qual inclui atos praticados dentro de casa por qualquer pessoa que conviva no espaço doméstico, como outros membros da família, cuidadores, empregados, sem função parental obrigatória (BRASIL, 2018). Esses tipos de violência estão frequentemente relacionados às agressões contra crianças e adolescentes.

De acordo com relatório emitido pela "United Nations Children's Fund" (UNICEF, 2017), três quartos das crianças com idade entre dois a quatro anos em todo o mundo, cerca de 300 milhões, são submetidos à disciplina violenta por pais e cuidadores, e, entre estas, 250 milhões são submetidas a punições físicas, sendo a violência responsável pela morte de uma criança ou adolescente a cada sete minutos no mundo. O cenário, no Brasil, corrobora com os dados estatísticos apresentados à nível mundial, sendo notável a replicação desse padrão de resultados.

De acordo com dados extraídos do Sistema Nacional de Agravos de Notificação (SINAN, 2017), diariamente são notificadas aproximadamente 233 agressões de diferentes tipos contra crianças e adolescentes de até 19 anos, e mais de 126 mil notificações de violência a indivíduos entre 0-19 anos foram registradas em 2017 em território nacional.

Devido a esse crescente número no Brasil, diversas medidas foram estabelecidas para tentar garantir a identificação e a redução desses casos. Dentre elas, citam-se a criação do Sistema de Vigilância de Violências e Acidentes (Viva) pelo Ministério da Saúde, e a instituição da notificação compulsória de violências no âmbito da saúde (ASSIS SG, et al., 2012). Acrescenta-se o estabelecimento de que qualquer tipo de violência infantojuvenil deva ser obrigatoriamente comunicado ao Conselho Tutelar, em uma tentativa de garantir 0 cumprimento do ECA.

O artigo 5 do ECA afirma que crianças e adolescentes, em hipótese alguma, devam ser objetificadas, tratadas sob qualquer perspectiva de violência, seja ela em forma de negligência, discriminação, exploração, opressão ou omissão, sendo sujeito passível de punição legal em casos de qualquer atentado a esses direitos (BRASIL, 2019a). 
Em 2011, estimava-se que apenas $20 \%$ das ocorrências eram realmente denunciadas (ACIOLI RML, et al., 2011). Assim, é notável que a subnotificação dos casos de violência infanto-juvenil permanece altamente prevalente, principalmente as intrafamiliares e as domésticas.

Associado a isso, estudos apresentam as falhas e as dificuldades estruturais na identificação e na notificação dos casos, as quais são feitas majoritariamente de maneira casual, sem sistematização (ROLIM ACA, et al., 2014), em grande parte pelo despreparo dos profissionais de saúde para manejar a violência, pelo desconhecimento acerca dos protocolos de notificação, e pelo receio de se confrontar e abordar o agressor (SALIBA O, et al., 2007).

Dessa forma, elucida-se a gravidade da subnotificação, que concede invisibilidade ao problema da violência infanto-juvenil, visto que a ausência de dados fidedignos falseia os reais números de vítimas, e interfere diretamente na atenção dedicada à elaboração de estratégias eficazes para combater o problema.

\section{A Violência no Contexto do Distanciamento Social}

Os casos de subnotificação, bem como a ocorrência de violência doméstica em geral, incluindo as agressões contra crianças e adolescentes, parecem ter se intensificado desde a adoção do regime de distanciamento social como estratégia de combate à Pandemia de Covid-19.

A OMS estima que cerca de 1,3 bilhões de crianças estejam afastadas das escolas, sem acessos a atividades em grupo, com visitas reduzidas aos consultórios médicos, restritas ao convívio familiar em domicílio, em um contexto de estresse, de medo, de excesso de informação midiática, de ameaça iminente de redução de renda, de incerteza sobre o futuro (CLUVER L, et al., 2020).

Assim, sujeitar as crianças e os adolescentes, já vulneráveis, ao contato exclusivo com a família por um período tão extenso e conturbado pode tornar o ambiente de casa um local de medo e de abuso, no qual o indivíduo vulnerável permanece mais tempo em contato com o agressor.

Essa situação é ainda mais intensificada entre famílias de baixa renda, residentes em moradias superlotadas e com poucos cômodos, fato que amplia ainda mais o convívio entre os membros durante a pandemia (MARQUES ES, et al., 2020).

Esse cenário é agravado pela ausência de supervisão ou contato com o meio externo, como educadores, colegas, médicos e outros profissionais de saúde, que garantem oportunidades rotineiras de identificação de sinais indicativos da necessidade de intervenção (GREEN P, 2020). Há, então, a redução do acesso aos sistemas de apoio públicos e institucionais, como escolas, creches, igrejas, o que dificulta a busca por ajuda e proteção.

Além disso, é importante ressaltar que esse intenso convívio domiciliar envolve ainda a sobrecarga dos pais, que precisam conciliar os cuidados da casa com os próprios trabalhos e com a atenção integral aos filhos, em meio ao conturbado cenário mundial.

Esta situação demanda adequação da dinâmica familiar mediante um contexto atípico, com limitações de mobilidade, de interação social e modificações das atividades rotineiras que precisaram ser interrompidas, contribuindo para quadros de maior irritabilidade não só nos pais, mas também nos filhos (ALIANÇA PARA A PROTEÇÃO DA CRIANÇA EM AÇÕES HUMANITÁRIAS, 2020).

Essa nova rotina familiar tem sido, dessa maneira, responsável por gerar conflitos diversos. Como consequência disso, acredita-se que as próprias crianças e adolescentes manifestam alguns comportamentos agressivos e desobedientes relacionados ao nível de estresse em que estão submetidas (MARQUES ES, et al., 2020).

Esse cenário não é completamente novo, visto haver registros similares da elevação da ocorrência da violência em contextos de distanciamento social anteriormente. Desse mesmo modo, segundo a UNICEF (2020), houve aumento das taxas de abuso e de exploração infanto-juvenil durante a epidemia do Ebola na África Ocidental, com maior registro de trabalho infantil, de negligência e de gravidez na adolescência, coincidente com períodos de fechamento das escolas. 
Na tabela abaixo, foram listadas as principais causas e riscos aos quais esses indivíduos estão submetidos durante a pandemia de Covid-19, com prejuízo das ações protetivas infanto-juvenis também observadas em surtos infecciosos anteriores (Quadro 1).

Quadro 1 - Riscos para a proteção da criança

\begin{tabular}{|c|c|}
\hline Causa do risco & $\begin{array}{l}\text { Risco apresentado pelo distanciamento na } \\
\text { pandemia de COVID-19 }\end{array}$ \\
\hline \multicolumn{2}{|c|}{ Maus-tratos Físicos e Mentais } \\
\hline $\begin{array}{l}\text { Distanciamento de escolas, das atividades } \\
\text { externas e em grupo, dificuldade de acesso } \\
\text { aos sistemas de apoio público e } \\
\text { institucionais } \\
\text { - Aumento do estresse psicológico entre } \\
\text { membros da família, incluindo pais, filhos e } \\
\text { outros } \\
\text { - Mau uso e acesso facilitado a produtos } \\
\text { tóxicos pelos pais e pelos filhos } \\
\text { - Aumento do tempo de permanência em } \\
\text { casa }\end{array}$ & $\begin{array}{l}\text { - Redução da supervisão e aumento da } \\
\text { negligência contra crianças e adolescentes } \\
\text { - Aumento dos conflitos familiares } \\
\text { - Aumento de abuso infanto-juvenil e } \\
\text { violência interpessoal/doméstica } \\
\text { - Envenenamento e outros perigos e riscos } \\
\text { de ferimentos de crianças autoprovocados, } \\
\text { acidentais ou provocados por terceiros } \\
\text { - Dificuldade de acesso aos serviços de } \\
\text { proteção } \\
\text { - Mais tempo de exposição a possíveis } \\
\text { desavenças e potenciais agressões }\end{array}$ \\
\hline \multicolumn{2}{|c|}{ Abuso Sexual } \\
\hline $\begin{array}{l}\text { - Redução na proteção familiar } \\
\text { - Imposição de responsabilidades domésticas, } \\
\text { como o cuidado de membros da família ou a } \\
\text { realização de tarefas domésticas } \\
\text { - Aumento de obstáculos para reportar } \\
\text { incidentes e para procurar tratamento } \\
\text { médico e outros serviços } \\
\text { - Aumento do tempo de permanência em } \\
\text { casa }\end{array}$ & $\begin{array}{l}\text { - Aumento do risco de exploração sexual de } \\
\text { crianças e adolescentes, incluindo sexo em } \\
\text { troca de ajuda } \\
\text { - Aumento da procura ou falta de acesso a } \\
\text { serviços de proteção da criança e do } \\
\text { adolescente } \\
\text { - Mais tempo de exposição a possíveis } \\
\text { desavenças e potenciais agressões } \\
\text { - Gravidez na adolescência }\end{array}$ \\
\hline \multicolumn{2}{|c|}{ Trabalho Infantil } \\
\hline $\begin{array}{l}\text { - Perda ou redução da renda familiar } \\
\text { - Oportunidade ou expectativa de trabalhar } \\
\text { - Ievido ao fechamento das escolas } \\
\text { Imposição de responsabilidades domésticas } \\
\text { às crianças e aos adolescentes, como } \\
\text { cuidado de membros da família ou a } \\
\text { realização de tarefas domésticas }\end{array}$ & $\begin{array}{l}\text { - Aumento do envolvimento da criança e do } \\
\text { adolescente em trabalho perigoso ou } \\
\text { explorador } \\
\text { - Exposição a agentes químicos, } \\
\text { aumentando risco de intoxicações } \\
\text { - Aumento do risco de contaminação da } \\
\text { criança e do adolescente por doenças } \\
\text { infecto contagiosas, como o COVID-19, } \\
\text { devido à maior exposição em trabalhos que } \\
\text { rompem o distanciamento social }\end{array}$ \\
\hline \multicolumn{2}{|c|}{ Negligência } \\
\hline $\begin{array}{l}\text { - Aumento do período de permanência da } \\
\text { criança e do adolescente dentro de casa, } \\
\text { associado ao aumento de convívio com os } \\
\text { pais e outros moradores, e à sobrecarga de } \\
\text { tarefa destes }\end{array}$ & $\begin{array}{l}\text { Incapacidade de atender à totalidade das } \\
\text { demandas dos filhos por parte dos pais, } \\
\text { uma vez que é necessário continuar o } \\
\text { trabalho e o cuidado da casa, mas perdem } \\
\text { a rede de apoio aos cuidados dos filhos } \\
\text { devido ao distanciamento, como escolas e } \\
\text { outras atividades extracurriculares } \\
\text { Designação de tarefas domésticas ou } \\
\text { paternas aos filhos devido à sobrecarga } \\
\text { dos pais }\end{array}$ \\
\hline
\end{tabular}

Fonte: Rita ACMS, et al., 2020. Dados extraídos de Aliança para a Proteção da Criança em Ações Humanitárias, 2020. 


\section{Consequências da Violência Para a Criança}

Quando se trata de violência doméstica, as crianças e os adolescentes podem ser vítimas diretas, na qual as agressões são voltadas especificamente a eles, e indiretas, nos casos em que presenciam a violência sofrida por outra pessoa. Em ambas as situações, são observados prejuízos na saúde física e mental destes que podem ocorrer a curto e em longo prazo (ALMEIDA A, et al., 2013).

Existem evidências de que a exposição à adversidade nos primeiros cinco anos de vida possa ter efeitos duradouros na maturação cerebral, visto que é um período sensível da vida, no qual as interações com os principais cuidadores são vitais para a aprendizagem e para a formação da criança (MUELLER I e TRONICK E, 2019). Sabe-se que a população infanto-juvenil está em processo de desenvolvimento físico e psíquico, portanto, sofrer atos de violência repercute direta e indiretamente no desempenho escolar, na adaptação social e no desenvolvimento orgânico da vítima.

Muitos desses indivíduos tornam-se mais agressivos na escola, o que prejudica a relação com colegas e professores (ALMEIDA A, et al., 2013), e costumam apresentar déficit no desempenho das habilidades de escrita e de leitura quando comparadas às crianças e aos adolescentes não vitimizados (BARROS $A$ e FREITAS M, 2015).

Além disso, de forma geral, crianças e adolescentes expostos à violência podem ter problemas futuros nas esferas social, emocional, cognitiva e física (ALMEIDA A, et al., 2013). Dentre as consequências emocionais em curto prazo, é possível citar pesadelos repetitivos, raiva, culpa, vergonha, medo, isolamento social, quadros fóbico-ansiosos e depressivos agudos. Esses indivíduos também são mais suscetíveis ao desenvolvimento, em longo prazo, de transtornos psiquiátricos, assim como de doenças psicossomáticas (ALMEIDA A, et al., 2013; REIS DM, et al., 2018).

Ademais, pode-se afirmar que a saúde mental está intimamente ligada à relação familiar, já que o risco de adolescentes apresentarem problemas mentais é cerca de três vezes maior quando a violência ocorre em âmbito familiar em comparação à violência urbana (BARROS A e FREITAS M, 2015). Dessa maneira, concluise que um bom relacionamento familiar é fundamental para a manutenção da saúde mental. Pesquisas apontam também que mulheres com história de violência na infância apresentam predisposição à vitimização em relacionamentos conjugais na vida adulta, enquanto homens nessa situação tendem a se envolver em relacionamentos amorosos como perpetuadores da violência, utilizando-a como método para resolução de conflitos (REIS DM, et al., 2018).

Já em relação às consequências físicas que a violência infanto-juvenil pode gerar, é possível citar como principais agravos às lesões e os hematomas localizados em diversas partes do corpo, os quais frequentemente passam despercebidos, principalmente após traumas leves. A exposição à violência em longo prazo pode causar cegueira, retardo mental, comprometimento neurológico e hepático (BARROS A e FREITAS M, 2015), e, apesar de menos prevalente, até homicídios. De acordo com a UNICEF (2020), no período de 1997 a 2017, cerca de 191 mil crianças e adolescentes de 10 a 19 anos foram vítimas de homicídio do Brasil.

\section{Como Suspeitar da Violência}

De acordo com a Sociedade Brasileira de Pediatria (SBP, 2001), na maioria das vezes, as vítimas não possuem evidências físicas de maus tratos, assim, a anamnese é fundamental no esclarecimento dos casos suspeitos de violência contra crianças e adolescentes. Contudo, diante do cenário atípico atual, há redução do contato com o profissional da saúde, sendo, então, de extrema importância à identificação precoce da violência doméstica infanto-juvenil como repercussão do distanciamento social.

Existem aspectos específicos que podem indicar a ocorrência de violência física, como a presença de lesões inexplicadas em lugares incomuns, o desenvolvimento de comportamento agressivo ou apático, irritabilidade frequente, choros sem motivo aparente, falta de autoestima e distúrbios do sono. Além disso, a desnutrição também pode acompanhar essas situações e acarretar o atraso no desenvolvimento da criança, evidenciando também a negligência familiar (SOCIEDADE DE PEDIATRIA DE SÃO PAULO e SOCIEDADE BRASILEIRA DE PEDIATRIA, 2018). 
No que tange aos maus tratos físicos, deve-se atentar à incompatibilidade da história relatada com as lesões existentes, e destas com o estágio de desenvolvimento do indivíduo. No caso dos profissionais de saúde, é preciso observar possíveis discordâncias entre os relatos do responsável para com os diferentes profissionais e as entrevistas individuais com a vítima e com o cuidador, atentando-se a supostos acidentes que ocorrem de forma repetitiva e com frequência além da esperada.

Ademais, os indivíduos que compõem a rede de apoio da vítima, como vizinhos, familiares e amigos devem estar atentos a possíveis indicativos de violência infanto-juvenil, expressos através de mudanças no padrão de comportamento da criança ou do adolescente. Entre essas, destacam-se alterações bruscas de humor, agressividade, vergonha excessiva, medo ou pânico, proximidade exagerada com determinada pessoa e regressão repentina a comportamentos infantis que já haviam sido abandonados (SOCIEDADE BRASILEIRA DE PEDIATRIA, 2001).

Além disso, também podem ser sugestivos de abuso a adoção de comportamentos sexualizados e o grande interesse por questões sexuais, assim como a presença de traumatismos físicos, infecções sexualmente transmissíveis, gravidez e enfermidades psicossomáticas (PAVÃO MTB, 2013).

Com relação aos maus tratos psicológicos, sabe-se estes são o tipo de violência mais difícil de ser detectada e costuma ocorrer concomitantemente a outros tipos de abusos. Assim, não há sinais específicos ou patognomônicos de que o indivíduo esteja sofrendo com isso, mas há algumas características que indicam maior necessidade de atenção para com a criança ou o adolescente.

Deve-se, portanto, atentar para antecedentes de violência e de ruptura familiar, número elevado de filhos, gravidezes não desejadas, mães adolescentes sem suporte psicossocial ou em situação de isolamento, distanciamento social, antecedentes psiquiátricos, responsáveis toxicômanos e dinâmica familiar instável, pois sabe-se que a violência é mais comum nos lares onde as relações familiares são precárias (SOCIEDADE BRASILEIRA DE PEDIATRIA, 2001).

Desse modo, evidencia-se que zelar pelas crianças é uma tarefa de todos, ou seja, dos profissionais de saúde, dos familiares e da comunidade. Assim, é de extrema importância que parentes mais próximos e vizinhos monitorem as crianças e os adolescentes, a fim de identificar os sinais de alerta de ocorrência de violência infanto-juvenil, sobretudo barulhos de agressão, atitudes estranhas da família e inadequação comportamental do indivíduo (PAVÃO MTB, 2013). A partir disso, torna-se possível o reconhecimento da ocorrência das agressões, possibilitando a denúncia e a proteção das crianças e dos adolescentes diante do cenário atual.

\section{Mobilização em Época de Pandemia}

Neste momento de distanciamento social, é importante ainda mais sensibilidade para identificar alguns sinais que possam remeter à necessidade de ajuda por parte das vítimas (REIS DM, et al., 2018). Dessa forma, percebe-se que o cuidado e a vigilância às crianças e aos adolescentes vítimas de agressão devem ser constantes.

Em relação a isso, uma das principais dificuldades consiste na construção de redes que priorizem as medidas preventivas, e tem sido crucial a ação conjunta de vários setores para se atingir o objetivo desejado (EGRY EY, et al., 2018). Assim, evidencia-se a importância adquirida pelas redes de atendimento que proporcionam formas de identificar e de reduzir, ou mesmo de erradicar, as agressões praticadas contra esses indivíduos (MARTINS CBG e JORGE MHPM, 2009), situação essa ainda mais delicada no contexto do distanciamento social.

Nesse cenário, a prevenção depende da ampla conscientização social acerca da violência intrafamiliar e doméstica. Portanto, tem-se investido na divulgação sobre o assunto, por meio da mídia e de outros veículos, para atentar as pessoas e capacitar o reconhecimento e a notificação desses casos (SANTOS ACW, 2006). Para isso, essas redes de proteção têm usado a internet aliada aos canais de denúncia como recurso fundamental para a propagação de informações, por exemplo, pelas diversas plataformas de comunicação social, portais digitais e aplicativos de denúncia criados em parceria com instituições reconhecidas, como a UNICEF e a Childhood Brasil (BRASIL, 2018). 
Essas denúncias de casos de violência contra a criança e ao adolescente podem ser realizadas por qualquer indivíduo que reconheça a situação de agressão, seja esta física, verbal, psicológica ou negligência. Reitera-se que as informações para identificação e o conhecimento dos canais de denúncia devem ser acessíveis a familiares, vizinhos e pessoas próximas, para que estejam atentos caso notem alguma mudança do comportamento e da fisionomia, barulhos, gritos, choros ou situações suspeitas envolvendo crianças e adolescentes (WEBER LND, et al., 2002).

Ainda nesse contexto, a Assembleia Legislativa de Minas Gerais (2020) aprovou o PL 1.054/19 que define como obrigatória a denúncia aos órgãos públicos de segurança de qualquer suspeita de violência nas dependências comuns e privadas dos condomínios residenciais.

Ressalta-se também a importância da participação da própria criança ou adolescente nesse processo, a começar pelo acesso ao conhecimento. De acordo com o artigo 53 do ECA, toda criança e adolescente deve ter assegurado o acesso à educação que garanta a ela o desenvolvimento pessoal, o reconhecimento dos princípios da cidadania e a construção do juízo e do senso de responsabilidade moral e social (BRASIL, 2019a). Nesse sentido, é essencial que o indivíduo compreenda a respeito da importância dos direitos humanos e das liberdades fundamentais, sendo estes aplicados à própria vida, criando ciência da forma correta que deve ser tratado, e do direito sobre o próprio corpo.

Dessa forma, por meio do desenvolvimento crítico, a criança ou o adolescente passa a ser participativo e protagonista no processo de reconhecimento e de prevenção de violências praticadas contra si, devendo estes se expressarem e serem ouvidos (ARANTES EMM, 2012).

Portanto, é fundamental que a prevenção e a denúncia da violência infanto-juvenil envolva tanto os adultos quanto as crianças e adolescentes, já que o combate à essa forma de agressão é caracterizado como dever de qualquer cidadão, principalmente pelo fato de as vítimas nem sempre serem capazes de se defenderem sozinhas, precisando frequentemente recorrer à ajuda (MATOSO MBL, et al., 2014).

\section{CONSIDERAÇÕES FINAIS}

O distanciamento social afasta crianças e adolescentes de sujeitos protetores, responsáveis por identificar fatores de risco e exposição à violência, e os aproxima de tensões advindas do intenso convívio familiar. Dessa forma, é necessário que a vigilância se intensifique e a atenção seja voltada para qualquer sinal de socorro que as vítimas possam emitir. Ressalta-se que o ECA torna dever zelar pela proteção das crianças e dos adolescentes que, em nenhuma hipótese, devem ser submetidas a qualquer perspectiva de violência, de forma que essa função recaia sobre todos os cidadãos devendo, estes, assumir esse papel. Assim, os sistemas de apoio multidisciplinares devem se estruturar e focar em medidas preventivas que abranjam desde a conscientização social sobre a identificação e a obrigatoriedade da denúncia, passando pelo protagonismo da criança ou do adolescente, até a sistematização dos protocolos de investigação e de notificação.

\section{REFERÊNCIAS}

1. ACIOLI RML, et al. Violência intrafamiliar contra crianças e adolescentes: identificação, manejo e conhecimento da rede de referência por fonoaudiólogo em serviços públicos de saúde. Rev. Bras. Saúde Mater. Infant., 2011; 11(1): 21-28.

2. ALIANÇA PARA A PROTEÇÃO DA CRIANÇA EM AÇÕES HUMANITÁRIAS. 2020. In: UNICEF. Nota Técnica: Proteção da Criança durante a Pandemia do Coronavírus.

3. ALMEIDA A, et al. Violência doméstica/intrafamiliar contra crianças e adolescentes: uma revisão bibliométrica. Rev. Interinst. Psicol., 2013; 6(2).

4. ARANTES EMM. Direitos da criança e do adolescente: um debate necessário. Psic. Clin., 2012; 24(1): 45-56.

5. ASSEMBLEIA LEGISLATIVA DE MINAS GERAIS. 2020. In: Assembleia Legislativa de Minas Gerais. Nova lei obriga síndico a comunicar violência doméstica.

6. ASSIS SG, et al. Notificações de violência doméstica, sexual e outras violências contra crianças no Brasil. Ciência \& Saúde Coletiva, 2012; 17(9): 2305-2317.

7. BARROS A, FREITAS M. Violência doméstica contra crianças e adolescentes: consequências e estratégias de prevenção com pais agressores. Pensando fam, 2015; 19(2).

8. BRASIL. Constituição da República Federativa do Brasil. Senado Federal, 1988.

9. BRASIL. Estatuto da Criança e do Adolescente. Senado Federal, 2019a. 
10. BRASIL. Ministério da Saúde. Secretaria de Vigilância em Saúde. Vigilância em saúde no Brasil 2003|2019: da criação da Secretaria de Vigilância em Saúde aos dias atuais. Bol Epidemiológico, 2019b; 50: 1-154.

11. BRASIL. Ministério dos Direitos Humanos. Violência contra Crianças e Adolescentes: Análise de Cenários e Propostas de Políticas Públicas. Ministério dos Direitos Humanos, 2018.

12. CLUVER L, et al. Parenting in a time of COVID-19. Lancet, 2020; 395(10231)

13. DAHLBERG LL, KRUG EG. Violência: um problema global de saúde pública. Ciência \& Saúde Coletiva, 2007; 11(sup): 1163-1178.

14. EGRY EY, et al. Notificação da violência infantil, fluxos de atenção e processo de trabalho dos profissionais da Atenção Primária em Saúde. Ciênc. Saúde colet., 2018; 23(1).

15. GREEN P. Risks to Children and Young People During covid-19 Pandemic. BMJ, 2020; 369.

16. MARQUES ES, et al. Violence against women, children, and adolescents during the COVID-19 pandemic: overview, contributing factors, and mitigating measures. Cad. Saúde Pública, 2020; 36(4).

17. MARTINS CBG, JORGE MHPM. A violência contra crianças e adolescentes: características epidemiológicas dos casos notificados aos Conselhos Tutelares e programas de atendimento em município do Sul do Brasil, 2002 e 2006. Epidemiol. Serv. Saúde, 2009; 18(4): 315-334.

18. MATOSO MB, et al. Violência intrafamiliar contra criança e adolescente: o papel do profissional de enfermagem e serviço social. Cadernos Brasileiros de Saúde Mental, 2014; 6(13): 73-89.

19. MUELLER I, TRONICK ED. Early Life Exposure to Violence: Developmental Consequences on Brain and Behavior. Front. Behav. Neurosci, 2019.

20. NUNES AJ, SALES MCV. Violência contra criança no cenário brasileiro. Ciência \& saúde coletiva, 2016; 21 (3): 871 880.

21. PAVÃO MTB. O impasse na suspeita ou na confirmação de abuso sexual infantil: a necessidade de encontrar marcas físicas. BIS, Bol. Inst. Saúde, 2013; 14(3), 264-179.

22. REIS DM, et al. O impacto da violência intrafamiliar no desenvolvimento psíquico infantil. Psicologia.pt, 2018.

23. ROLIM ACA, et al. Subnotificação de maus-tratos em crianças e adolescentes na Atenção Básica e análise de fatores associados. Saúde Debate, 2014; 38(103): 794-804.

24. SALIBA O, et al. Responsabilidade do profissional de saúde sobre a notificação de casos de violência doméstica. Rev. Saúde Pública, 2007; 41(3): 472-477.

25. SANTOS ACW. Caminhos para combate e prevenção de violência intrafamiliar. Paidéia (Ribeirão Preto), 2006; 16(35): 437-439.

26. SILVA, MJS; et al. O conceito de saúde na Saúde Coletiva: contribuições a partir da crítica social e histórica da produção científica. Revista de Saúde Coletiva, 2019; 29(1).

27. SISTEMA DE INFORMAÇÕES SOBRE AGRAVOS DE NOTIFICAÇÃO. 2017. In: SINAN. Disponível em: portalsinan.saude.gov.br/. Acesso em: 27 maio 2020.

28. SOCIEDADE BRASILEIRA DE PEDIATRIA. Guia de Atuação frente a maus-tratos na infância e na adolescência: orientações para pediatras e demais profissionais que trabalham com crianças e adolescentes, 2001; 2 ed.

29. SOCIEDADE DE PEDIATRIA DE SÃO PAULO, SOCIEDADE BRASILEIRA DE PEDIATRIA. Manual de atendimento às crianças e adolescentes vítimas de violência. Conselho Federal de Medicina, 2018; 2.ed.

30. STEFANINI JR, et al. Violência intrafamiliar e as repercussões para a saúde da mulher: compreendendo a história de Antônia. Rev. NUFEN, 2019; 11(1): 122-136.

31. UNITED NATIONS CHILDREN'S FUND. 2020. In: UNICEF. Covid-19: Crianças em risco aumentado de abuso, negligência, exploração e violência em meio a intensificação das medidas de contenção.

32. UNITED NATIONS CHILDREN'S FUND. A Familiar Face: Violence in the lives of children and adolescents, 2017. UNICEF, 2017.

33. WEBER LND, et al. Famílias que maltratam: uma tentativa de socialização pela violência. Psico-USF (Impr.), 2002; $7(2): 163-173$. 